\title{
Reduction of shock wave lithotripsy-induced renal tubular injury by tadalafil
}

\author{
Danisoglu $\mathrm{ME}^{1}$, Aytac $\mathrm{B}^{2}$, Kilicaslan $\mathrm{H}^{1}$, Dogan $\mathrm{S}^{1}$, Vuruskan $\mathrm{H}^{1}$ \\ Uludag University, Faculty of Medicine, Department of Surgical Pathology, Gorukle, Bursa, Turkey. \\ bernaaytac@uludag.edu.tr
}

\begin{abstract}
Objectives: The aim of the study was to examine whether administration of tadalafil, a phosphodiesterase type 5 inhibitor, has a protective effect in the prevention of renal injury in a rat model after Shock Wave Lithotripsy (SWL), with the assessment based on histopathologic examination and measurement of Heat Shock Protein 70 expression.

Methods: A total of 40 adult, male Sprague-Dawley rats were divided into five groups as follows; control group, group SN3, group SN7, group TSN3, TSN7. Both kidneys were evaluated regarding tubular damage, peritubular fibrosis and glomerular damage using light microscopy. We examined HSP-70 expression, which occurred in response to renal ischemic injury observed after SWL. The groups were compared between each other and with the control group.

Results: No statistically significant difference was found when the groups were compared using light microscopy for the changes in glomeruli. Tubular necrosis, loss of microvilli and peritubular fibrosis were less in Group TSN3 and Group TSN7 compared to Group SN3. Similarly, tubular necrosis, loss of microvilli and peritubular fibrosis were less in Group TSN3 and Group TSN7 compared to Group SN7. HSP-70 staining was less in Group TSN3 and Group TSN7 compared to Group SN3 and Group SN7.

Conclusion: Based on the results of light microscopy and HSP-70 staining, we demonstrated that SWL could cause renal ischemia- reperfusion injury. Our results suggested that Tadalafil administration could prevent this SWL-related renal cell injury (Tab. 2, Fig. 5, Ref. 28). Text in PDF www.elis.sk.

Key words: heat shock protein, shock wave lithotripsy, ischemia-reperfusion injury, tadalafil, renal tubules.
\end{abstract}

Shockwave lithotripsy (SWL) has been proven to be an effective method in the management of urinary stone disease (1). However clinical and experimental studies have demonstrated that renal and extrarenal side effects can occur as a consequence of SWL (2). The possible cause of alterations in kidney is due to renal vasoconstriction and intraparenchymal bleeding that occurs in kidneys after SWL. Hypoxic tissue can be seen around sites of intraparenchymal bleeding (3). These findings suggest that oxidative stress mediated by ischemia-reperfusion (IR) might contribute to renal injury subsequently to SWL (3). Heat shock protein 70 (HSP 70) is an intracellular stress protein that was originally found to accumulate in cells after thermal stress, oxidative stress, infection, heavy metals, atherosclerosis, congestive heart failure, malignant, autoimmune diseases and radiation (4). Tadalafil (Td) is a class of mild vasoactive drugs developed for the treatment of erectile dysfunction (ED). It has a different chemical structure from sildenafil and vardenafil with a mean time to maximum plasma concentration of $2 \mathrm{~h}$ which is two

\footnotetext{
${ }^{1}$ Uludag University, Faculty of Medicine, Department of Urology, Bursa, Turkey, and ${ }^{2}$ Uludag University, Faculty of Medicine, Department of Surgical Pathology, Bursa, Turkey
}

Address for correspondence: B. Aytac, Uludag University, Faculty of Medicine, Department of Surgical Pathology, 16059, Gorukle, Bursa, Turkey.

Phone: +90.224 .2953690 , Fax: +90.224 .4428688$ times longer than other molecules $(5,6)$. These advanced features make this molecule a good option in the regulation of decreased vascular resistance and ischemia. It has been demonstrated that $\mathrm{Td}$ administration as a single dose, prior to renal I/R injury, attenuated these morphological disarrangements which were observed in renal $\mathrm{I} / \mathrm{R}$ (7). Our study aimed to assess renal trauma and inflammation after SWL by measuring renal parenchymal levels of HSP-70 expression and performing a histopathological examination. In addition we examined the preventive effect of $\mathrm{Td}$ for the renal $\mathrm{I} / \mathrm{R}$ injury in a rat model.

\section{Methods}

All experiments in this study were performed in accordance with the guidelines for animal research from the National Institutes of Health and were approved by our university Animal Ethics Committee (2010-01/03).

\section{Experimental groups}

Male Sprague-Dawley rats weighing 250-300 g were randomly divided into five groups each with eight animals. The rats were anesthetized with $1 \mathrm{mg} / \mathrm{kg}$ intramuscular injection of ketamine $\mathrm{HCl}$ and xylazine $\mathrm{HCl}(10 \mathrm{mg} / \mathrm{kg})$. All rats underwent laparotomy. An upper abdominal midline incision was made, in which two hemoclips were placed in the perirenal adipose tissue of both sides. SWL was given to all rats by focusing on the hemoclips except 


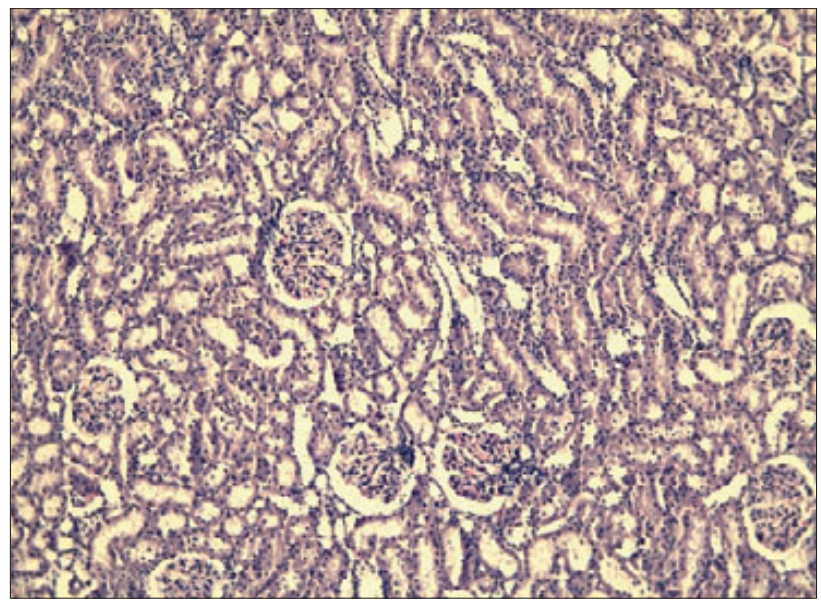

Fig. 1. Regular morphology of renal parenchyma in control group (hematoxylin-eosin x100).

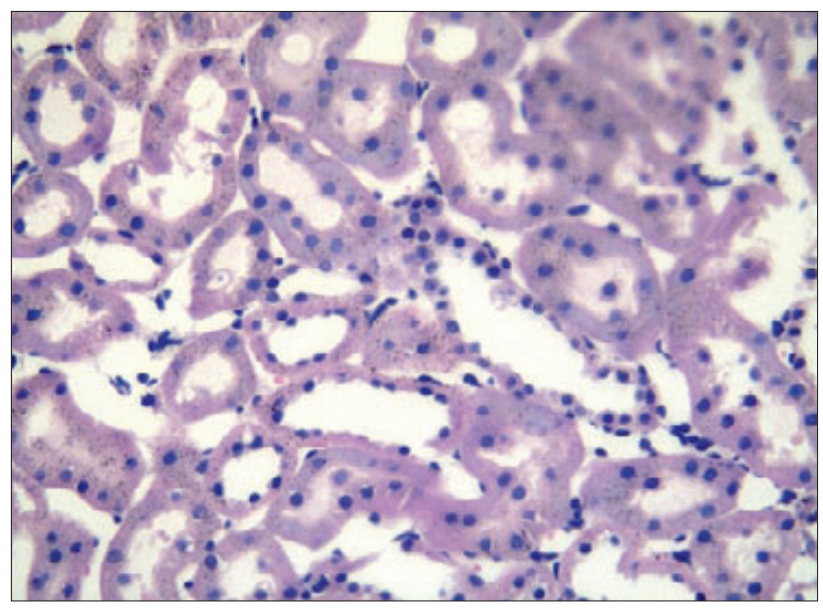

Fig. 2. Tubular necrosis and hyalinization in Group SN3 and Group SN7 (hematoxylin-eosin x200).

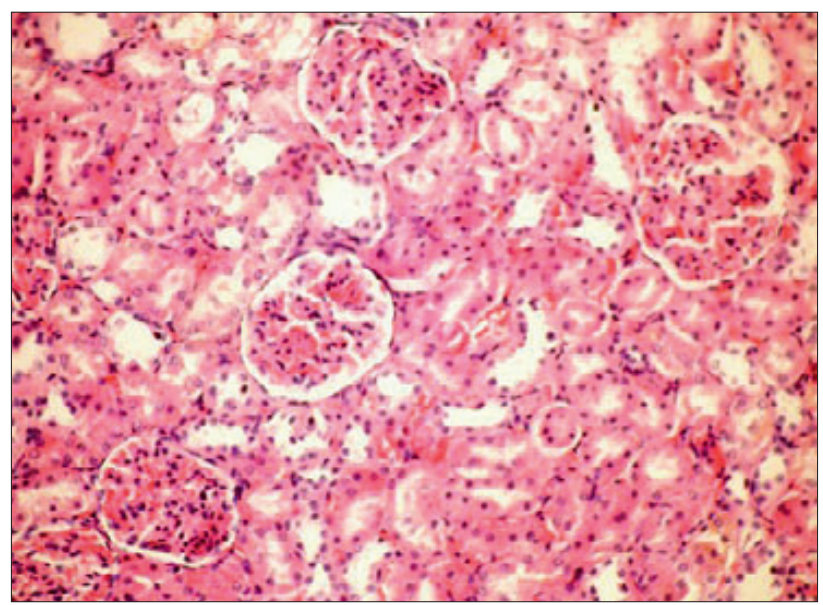

Fig. 3. Normal appearance of glomeruli and tubular cells in Group TSN3 (hematoxylin-eosin x200).

the control group. Td was dissolved in saline solution and given as a single dose $(1 \mathrm{mg} / \mathrm{kg})$ through an orogastric tube $150 \mathrm{~min}$ utes before the SWL in the Td pretreatment groups. The number

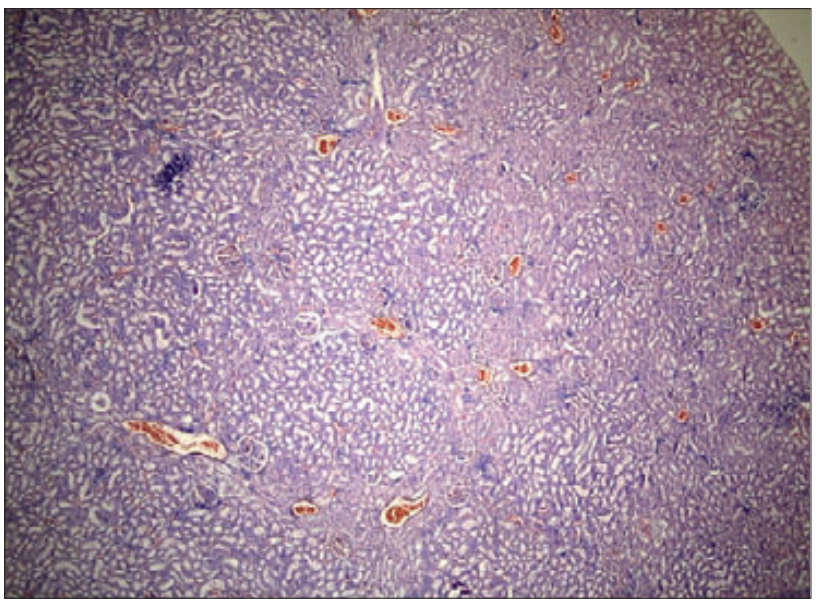

Fig. 4. Stasis, congestion and hemorrhage in some sections of cortex and medulla in Group TSN7 (hematoxylin-eosin x40).

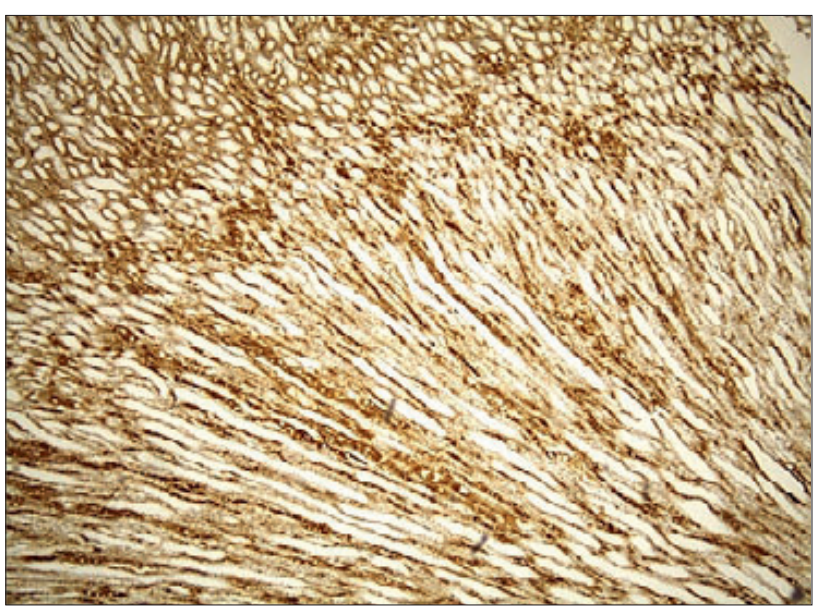

Fig. 5. HSP-70 staining of cortical and medullar collector tubules in Group SN3 and Group SN7.

of shock waves given was 1,500 SW per session and the energy setting was $14 \mathrm{kV}$ using Multimed Classic 2006, ELMED, Ankara/ Turkey. All rats underwent bilateral nephrectomy.

In the control group, bilateral nephrectomy was performed 24 hours after laparotomy. Animals in Group SN3 (SWL + nephrectomy at day 3), underwent bilateral nephrectomy 3 days after SWL. In Group SN7 (SWL + nephrectomy at day 7), bilateral nephrectomy was performed 7 days after SWL. In Group TSN3 (Td followed by SWL + nephrectomy at day 3), rats were given Td before SWL and 3 days later they underwent bilateral nephrectomy. In Group TSN7 (Td followed by SWL + nephrectomy at day 7), rats were given Td before SWL and 7 days later they underwent bilateral nephrectomy.

\section{Histopathological evaluation}

Both kidneys were fixed with formalin, embedded in paraffin and stained with hematoxylin eosin. All were evaluated by the same pathologist with respect to glomerular, tubular, and interstitial morphology. Sclerosis of glomeruli was noted. About one 
hundred tubules and 20 glomeruli were evaluated randomly and were scored for each section. The mean score was calculated for each of the five groups. Tubular morphology was determined in four groups as: 0 (normal morphology), 1 (mild brush border abnormality such as flattening, or irregularity in shape and size of the cells), 2 (moderate brush border abnormality, involving more than $50 \%$ of the tubular profiles), 3 (loss of microvilli in the epithelium of whole tubules and tubular necrosis).

For HSP-70 staining, the sections were deparaffinized in xylene and rehydrated in graded ethanol. The endogenous peroxidase was blocked by incubation in $1 \% \mathrm{H}_{2} \mathrm{O}_{2}$ solution in methanol for 5 minutes. The sections were boiled in $10 \mu \mathrm{M}$ sodium citrate $(\mathrm{pH}$ 6.0) for 10 minutes in a microwave oven, and then incubated for 30 minutes at room temperature with the primary antibody (hsp70 Ab.2, Clone W 27; Neo Markers). The reaction was visualized using an LSAB 2 kit and DAB chromogen (DAKO). Sections were then lightly counterstained with hematoxylin. Staining without primary antibody served as negative control. For evaluation of HSP-70 staining cytoplasmic immunoreactivity was accepted positive. Following evaluation of 10 high-power fields, the fractions of positive cells were recorded as 0 (negative), $1(<5 \%), 2(5 \%$ to $50 \%$ ), or $3(>50 \%)$ in tubules and glomeruli.

Statistical analysis was done using the Statistical Package for Social Sciences, ver. 13.0 for Windows, (SPSS, Chicago, IL). $\chi^{2}-$ test was used for analysis of proportions. The value of $p<0.05$ was considered statistically significant.

\section{Results}

Microscopic evaluation of the Group control revealed a regular morphology of renal parenchyma (Fig. 1). The kidneys in Group SN3 showed loss of micro- villi in the epithelium of proximal tubules. Tubular necrosis was found in both cortical and medullar regions (Fig. 2). Enlargement of the interstitiuml was seen as a result of fibrosis. Tubular degeneration was less evident in Group SN7 compared to Group SN3. Necrosis in the tubular epithelium of the cortex and hyaline degeneration in some areas were observed.
Interstitial fibrosis was prominent. In Group TSN3, capillaries in glomeruli and tubular cells have shown normal morphology (Fig. 3). Interstitial edema was seen, particularly near blood vessels and interstitial fibrosis was mild. In Group TSN7, the kidney preserved the normal morphology, and showed normal morphology of glomeruli and tubular cells. Most of the epithelial cells lining the proximal tubules were preserving microvilli structures. Stasis, congestion and hemorrhage in some sections were found in the medulla (Fig. 4).

When the groups were compared using light microscope for the changes of glomeruli, no statistically significant difference was found. Renal tubular damage and peritubular fibrosis was significantly increased in Group SN3 and Group SN7 when compared to the control group. Tubular necrosis, loss of microvilli and peritubular fibrosis were less in Group TSN3 and Group TSN7 compared to Group SN3. Similarly tubular necrosis, loss of microvilli and peritubular fibrosis were less in Group TSN3 and Group TSN7 compared to Group SN7 (Tab. 1).

In Group SN3, Group SN7, Group TSN3 and Group TSN7, HSP-70 staining in glomeruli was increased compared to control group but the difference was statistically insignificant (Tab. 2). Proximal tubule cells were not stained with HSP-70 in any of the groups. In cortical collector tubules, HSP-70 staining was significantly increased in Group SN3 and Group SN7 when compared to those in the control Group (Fig. 5). HSP-70 staining was lower in Group TSN3 and Group TSN7 compared to Group SN3. Similarly HSP-70 staining was lower in Group TSN3 and Group TSN7 compared to Group SN7. However, the difference between Group SN3 and Group SN7 was not significant. Also there was no difference between Group TSN3 and Group TSN7 regarding HSP-70 staining (Tab. 2).

In medullar collector tubules, HSP-70 staining was significantly increased in Group SN3 and Group SN7 when compared to those in the control Group. HSP-70 staining was lower in Group TSN3 and Group TSN7 compared to Group SN3. Similarly HSP70 staining was less in Group TSN3 and Group TSN7 compared to Group SN7. The difference between Group SN3 and Groups

Tab. 1. Comparison of groups between each other regarding the tubular damage and peritubular fibrosis. Numbers indicate the $p$ values of chi-square test which was utilized to compare two individual groups.

\begin{tabular}{|c|c|c|c|c|c|c|c|c|}
\hline & \multicolumn{4}{|c|}{ Tubular damage } & \multicolumn{4}{|c|}{ Peritubular fibrosis } \\
\hline & Group SN3 & Group SN7 & Group TSN3 & Group TSN7 & Group SN3 & Group SN7 & Group TSN3 & Group TSN7 \\
\hline Group sham & $<0.001$ & $<0.001$ & 0.144 & 1.000 & $<0.001$ & $<0.001$ & 0.144 & 1.000 \\
\hline Group SN3 & - & 0.365 & $<0.001$ & $<0.001$ & - & 0.365 & $<0.001$ & $<0.001$ \\
\hline Group SN7 & - & - & $<0.001$ & $<0.001$ & - & - & $<0.001$ & $<0.001$ \\
\hline Group TSN3 & - & - & - & 0.144 & - & - & - & 0.144 \\
\hline
\end{tabular}

Tab. 2. Comparison of groups between each other regarding HSP-70 staining of glomeruli. cortical and medullar collector tubules. Numbers indicate the $p$ values of chi-square test which was utilized to compare two individual groups.

\begin{tabular}{|c|c|c|c|c|c|c|c|c|c|c|c|c|}
\hline & \multicolumn{4}{|c|}{ Glomeruli } & \multicolumn{4}{|c|}{ Cortical collector tubules } & \multicolumn{4}{|c|}{ Medullar collector tubules } \\
\hline & Group & Group & Group & Group & Group & Group & Group & Group & Group & Group & Group & Group \\
\hline & SN3 & SN7 & TSN3 & TSN7 & SN3 & SN7 & TSN3 & TSN7 & SN3 & SN7 & TSN3 & TSN7 \\
\hline Group sham & 0.003 & 0.022 & 0.102 & 0.022 & $<0.001$ & $<0.001$ & 1.000 & 0.446 & $<0.001$ & $<0.001$ & 0.144 & 1.000 \\
\hline Group SN3 & - & 0.476 & 0.157 & 0.476 & - & 1.000 & $<0.001$ & $<0.001$ & - & - & $<0.001$ & $<0.001$ \\
\hline Group SN7 & - & - & 0.476 & 1.000 & - & - & $<0.001$ & $<0.001$ & - & - & $<0.001$ & $<0.001$ \\
\hline Group TSN3 & - & - & - & 0.476 & - & - & - & 0.446 & - & - & - & 0.144 \\
\hline
\end{tabular}


SN7 was not significant. Additionally, there was no difference between Group TSN3 and Group TSN7 regarding HSP-70 staining in medullar collector tubules (Tab. 2).

\section{Discussion}

The effects of SWL on renal morphology and renal function have been studied previously (8-10). According to these studies, the energy released by shock waves produces cavitations or bubbles that may cause direct injury to the renal vasculature. Vascular injury causes ischemia in renal tissue, which becomes susceptible to free-radical production as reperfusion occurs (1115 ). The $70-\mathrm{kDa}$ family of heat shock proteins (HSP70) plays a central role in cellular protection (4). In kidney, the 70-kDa HSP production localizes primarily in renal tubular cells (16). HSP70 has been associated with cytoprotection in response to several injuries, including oxidative stress, ischemia and nephrotoxicity (17). In renal epithelial cells exposed to injury, it was reported that changes in protein structure or conformation trigger the increase in HSP70 mRNA (18).

There are few experimental studies in the literature that report the relation of renal parenchymal heat shock protein 70 (HSP 70) levels and traumatic effects of SWL. While earlier studies focused on oxidative injury to the renal cortex (15), Clark et al showed that acute oxidative stress and inflammation after SWL are highly localized to the renal medulla within the focus (F2) of the lithotripter (19). In our study we also observed that cortical and medullar collector tubules were more affected than glomeruli. Sarica et al showed during early follow-up ( 24 hours) that all kidneys which were treated or untreated with SWL demonstrated moderate to severe HSP-70 positivity. They reported that assessment of tissue HSP70 levels during late follow-up (7 days) demonstrated moderate or limited degrees of positivity in the treated kidneys. Limited or no positivity could be demonstrated in the untreated kidneys during this period (20). In our study the groups which were treated with SWL and underwent nephrectomy at day 3 and 7 demonstrated no difference regarding HSP70 staining positivity. This finding shows that renal injury process still continued during the observation period of our study.

Oxygen-free radical production related to ischemia/reperfusion after SWL is believed to contribute to parenchymal damage via lipid peroxidation and the disruption of cellular and mitochondrial membranes, and cellular damage (21). There is evidence that biological responses triggered by oxidative products are associated with lipid peroxidation derivatives, which are able to induce various pathogenic intracellular signals involving calcium, G-proteins, cAMP, cGMP, phospholipase $\mathrm{C}$ and $\mathrm{D}$, protein kinase $\mathrm{C}$, ceramide, and MAP kinase cascade leading to cellular dysfunction (21).

According to these pathways various pharmacologic agents have been evaluated to preserve renal morphology and function during renal injury caused by SWL. Verapamil, an organ-protective calcium antagonist is one of these agents. It has an excellent stabilizing effect on post ischemic malperfusion, and has been found to be beneficial in this respect (22). Nifedipine exhibits a protective effect on shock-wave-induced tubular damage similar to verapamil (23). In a study from China the authors concluded that the preparation of traditional medicines for invigorating the kidney and excreting calculus may be of significant benefit in protecting the kidneys against SWL-induced morphological and functional injury. According to their results these preparations exert their effects by improving renal resistance to oxidative stress, ameliorating circulatory disorders, and interfering with local inflammation (12). Among these agents, PDE5 inhibitors currently in clinical use for erectile dysfunction have a protective effect on renal ischemia/reperfusion (I/R) injury $(24,25)$. It is specific for the hydrolysis of cGMP (26). cGMP plays an important role in regulation of intracellular calcium levels, increases the release of $\mathrm{NO}$, induces vasodilatation and improves endothelial dysfunction in I/R injury (26). They differ in their selectivity, efficacy, side effects and pharmacokinetics data. Our results revealed that rats treated with tadalafil, regardless of the day of the scarification, have similar features with control group and significantly improved findings when compared to untreated groups in terms of all parameters of renal damage (microscopic findings of tubular damage and peritubular fibrosis and HSP-70 staining). The independency of findings on the day of sacrification in the tadalafil treated group may be attributed to the longer half life of the drug which needs to be confirmed by comparative studies using other agents (27). The half-lives of sildenafil and vardenafil are $4 \mathrm{~h}$ and that of tadalafil is $17.5 \mathrm{~h}$. These advanced features make this molecule a good option in the treatment of regulation of decreased vascular resistance and ischemia (28). Although there are studies reporting protective effect of PDE5 inhibitors on renal $\mathrm{I} / \mathrm{R}$ injury, this is the first study investigating the protective effect of these agents in renal injury due to SWL treatment.

\section{Conclusion}

The results of our study have demonstrated that given as a single dose Td, prior to SWL decreases renal injury due to a vasoconstrictive effect. We obtained this result both by determining the damage in tubular cells with light microscopy and immunohistochemicaly by HSP70 staining. This promising preliminary study encourages further future clinical studies with a longer follow-up and including the comparison of various agents.

\section{References}

1. Porfyris OT, Cutress ML, Tolley DA. The use of extra-corporeal shockwave lithotripsy for obstructing ureteric stones. Minerva Urol Nefrol 2011; 63 (2): 175-182.

2. McAteer JA, Evan AP. The acute and long-term adverse effects of shock wave lithotripsy. Seminars Nephrol 2008; 28 (2): 200-213.

3. Sarica K, Kosar A, Yaman O, Bedük Y, Durak I, Gogus O, Kavukcu M. Evaluation of ischemia after ESWL: detection of free oxygen radical scavenger enzymes in renal parenchyma subjected to high-energy shock waves. Urol Int 1996; 57 (4): 221-223.

4. Aufricht C. Heat-shock protein 70: Molecular supertool? Pediatr Nephrol 2005; 20 (6): 707-713. 
$616-620$

5. Sesti C, Florio V, Johnson EG, Kloner RA. The phosphodiesterase-5 inhibitor tadalafil reduces myocardial infarct size. Int J Impot Res 2007; 19 (1): 55-61.

6. Guzeloglu M, Yalcinkaya F, Atmaca $S$ et al. The beneficial effects of tadalafil on renal ischemia-reperfusion injury in rats. Urol Int 2011; 86 (2): 197-203.

7. Gasanov F, Aytac B, Vuruskan H. The effects of tadalafil on renal ischemia reperfusion injury: an experimental study. Bosn J Basic Med Sci 2011; 11 (3): 158-162.

8. Ulu B, Tüzel E, Aktepe F, Akdoğan B, Güler C. Evaluation of shockwave lithotripsy induced renal damage using heat shock protein 70 expression in the presence of different urinary obstruction periods. J Endourol 2009; 23 (7):1093-1098.

9. De Stefani S, Micali S, De Carne $\mathbf{C}$ et al. Shockwave lithotripsy and protective role of inosine: early and late evaluation in an experimental model. J Endourol 2008; 22 (5): 1059-1063.

10. Sarica K, Yencilek F. Prevention of shockwave induced functional and morphological alterations: an overview. Arch Ital Urol Androl 2008; 80 (1): 27-33.

11. Al-Awadi KA, Kehinde EO, Loutfi I et al. Treatment of renal calculi by lithotripsy: minimizing short-term shock wave induced renal damage by using antioxidants. Urol Res 2008; 36 (1): 51-60.

12. Sheng B, He D, Zhao J et al. The protective effects of the traditional Chinese herbs against renal damage induced by extracorporeal shock wave lithotripsy: a clinical study. Urol Res 2011; 39 (2): 89-97.

13. Evan AP, Willis LR, Mcateer JA et al. Kidney damage and renal functional changes are minized by waveform control that suppresses cavitations in shock wave lithotripsy. J Urol 2002; 168 (4 Pt 1): 1556-1562.

14. Sheng BW, Chen XF, Zhao J, He DL, Nan XY. Astragalus membranaceus reduces free radical-mediated injury to renal tubules in rabbits receiving high-energy shock waves. Chin Med J (Engl) 2005; 118 (1): 43-49.

15. Delvecchio F, Auge BK, Munver R et al. Shock wave lithotripsy causes ipsilateral renal injury remote from the focal point: the role of regional vasoconstriction. J Urol 2003; 169 (4): 1526-1529.

16. Wakui H, Komatsuda A, Miura AB. Heat-shock proteins in animal models for acute renal failure. Ren Fail 1995; 17 (6): 641-649.
17. Donati YR, Slosman DO, Polla BS. Oxidative injury and the heat shock response. Biochem Pharmacol 1990; 40 (12): 2571-257.

18. Chen Q, Yu K, Stevens JL. Regulation of the cellular stress response by reactive electrophiles. The role of covalent binding and cellular thiols in transcriptional activation of the 70-kilodalton heat shock protein gene by nephrotoxic cysteine conjugates. J Biol Chem 1992; 267 (34): 24322-24327.

19. Clark DL, Connors BA, Evan AP, Willis LR, Handa RK, Gao S. Localization of renal oxidative stress and inflammatory response after lithotripsy. BJU Int 2009; 103 (11): 1562-1568.

20. Delius M, Jordan M, Eizenhoefer H et al. Biological effects of shock waves: kidney hemorrhage by shock waves in dogs--administration rate dependence. Ultrasound Med Biol 1988; 14 (8): 689-694.

21. Milani E, Nikfar S, Khorasani R, Zamani MJ, Abdollahi M. Reduction of diabetes-induced oxidative stress by phosphodiesterase inhibitors in rats. Comp Biochem Physiol C Toxicol Pharmacol 2005; 140 (2): 251-255.

22. Yaman O, Sarica K, Ozer G et al. Protective effect of verapamil on renal tissue during shock wave application in rabbit model. J Endourol 1996; 10 (4): 329-333.

23. Strohmaier WL, Koch J, Balk N, Wilbert DM, Bichler KH. Limitat Wright PJ. ion of shock-wave-induced renal tubular dysfunction by nifedipine. Eur Urol 1994; 25 (2): 99-104.

24. Lau LC, Adaikan PG. Mechanisms of direct relaxant effect of sildenafil, tadalafil and vardenafil on corpus cavernous. Eur J Pharmacol 2006; 541 (3): 184-190.

25. Kukreja RC, Ockaili R, Salloum F et al. Cardioprotection with phosphodiesterase-5 inhibition--a novel preconditioning strategy. J Mol Cell Cardiol 2004; 36 (2): 165-173.

26. Willams P, Lopez H, Britt D, Chan C, Ezrin A, Hottendorf R. Characterization of renal ischemia-reperfusion injury in rats. J Pharmacol Toxicol Methods 1997; 37: 1-7.

27. Bischoff E. Vardenafil preclinical trial data: potency, pharmacodynamics, pharmacokinetics, and adverse events. Int J Impot Res 2004; 16: 34-37.

28. Wright PJ. Comparison of phosphodiesterase type 5 (PDE5) inhibitors. Int J Clin Pract 2006; 60 (8): 967-975. 